\title{
Garantias basilares à saúde mental em cenário pandêmico: reflexões sobre as implicações psicológicas nos profissionais de saúde
}

\section{Basic guarantees for mental health in a pandemic scenario: reflections on psychological claims in health professionals}

Garantías básicas para la salud mental en un escenario pandémico: reflexiones sobre reclamos psicológicos en los profesionales de la salud

Deyse Janiele Bernardo Oliveira ORCID: https://orcid.org/0000-0002-1270-8431 Universidade Federal do Rio Grande do Norte, Brasil

E-mail: deyse.oliveiira@hotmail.com

José Isaul Pereira

ORCID: https://orcid.org/0000-0002-5027-0434 Universidade Federal do Rio Grande do Norte, Brasil

E-mail: isaulpsico@hotmail.com

Kelly Bezerra de Oliveira

ORCID: https://orcid.org/0000-0002-4499-1980 Universidade Federal do Rio Grande do Norte, Brasil

E-mail: kelly.bez.oliveira@ @otmail.com

Larissa Maria Souto Moura

ORCID: https://orcid.org/0000-0003-4592-4648 Universidade Federal do Rio Grande do Norte, Brasil E-mail: lari.maria247@gmail.com

Rejane Silva dos Santos

ORCID: https://orcid.org/0000-0001-7637-3856 Universidade Federal do Rio Grande do Norte, Brasil E-mail: rejannysantos32@gmail.com Aline Cristina Silva

ORCID: https://orcid.org/0000-0001-9659-9748 Universidade Federal do Rio Grande do Norte, Brasil E-mail: alinecristinas8@ hotmail.com

Francyely dos Santos Moura

ORCID: https://orcid.org/0000-0003-3791-2049

Universidade Federal do Rio Grande do Norte, Brasil E-mail: mourafrancyely@gmail.com

Hugo Sarmento Gadelha ${ }^{1}$

ORCID: https://orcid.org/0000-0001-9414-0554 Universidad del Museo Social Argentino, Argentina E-mail: hugoscurso@uol.com.br

José Cândido da Silva Nóbrega

ORCID: https://orcid.org/0000-0002-0976-3763

Universidade Federal de Campina Grande, Brasil E-mail: jcandidosn@uol.com.br

Manoel Marques de Souto Nóbrega Filho ORCID: https://orcid.org/0000-0003-4512-2733

Faculdade de Ciências Médicas da Paraíba, Brasil

E-mail: manoelmarquesnobrega @gmail.com

José Djalisson Santos Oliveira

ORCID: https://orcid.org/0000-0002-3335-1144

Centro Universitário de João Pessoa, Brasil E-mail: oliveira.eujose@gmail.com

\section{Resumo}

As condições de trabalho e a forma como o indivíduo atribui significado a este, poderá contribuir com danos severos à saúde mental. Considerando o cenário atual, os profissionais de saúde estão sujeitos diariamente a eventos estressores advindo do seu processo de trabalho. A pesquisa tem por objetivo analisar possíveis implicações na saúde mental dos

\footnotetext{
${ }^{1}$ Doutorando pela Universidad del Museo Social Argentino
} 
profissionais de saúde no cenário de pandemia pela COVID-19. Para tal, o presente estudo foi desenvolvido com base na pesquisa bibliográfica, amparando-se em pesquisas que abordassem a questão da saúde mental relacionado às condições de trabalho dos profissionais de saúde em tempos de pandemia. A partir da análise dos dados, pode-se constatar que o atual cenário e as demandas enfrentadas no seu âmbito de trabalho estabelecem-se como fatores contribuintes para o surgimento de danos emocionais e implicações psicológicas à saúde dos profissionais de saúde. Dessa maneira, os recursos de apoio que auxiliam no suporte psicológico das demandas vivenciadas por esses profissionais fazem-se essenciais nos serviços de saúde.

Palavras-chave: Saúde Mental; Pandemia; Profissionais da saúde; Impactos na saúde.

\begin{abstract}
The working conditions and the way in which the individual gives meaning to it, can contribute to severe damage to mental health. Considering the current scenario, health professionals are daily subject to stressful events arising from their work process. The research aims to analyze possible implications for the mental health of health professionals in the pandemic scenario caused by COVID-19. To this end, the present study was developed based on bibliographic research, supported by research that addressed the issue of mental health related toworking conditions of health professionals in times of pandemic. From the data analysis, it can be seen that the current scenario and the demands faced in their work environment are established as contributing factors for the emergence of emotional damage and psychological implications for the health of health professionals. Thus, the support resources that help in the psychological support of the demands experienced by these professionals are essential in health services.
\end{abstract}

Keywords: Mental Health; Pandemic; Health professionals; Health impacts.

\title{
Resumen
}

Las condiciones de trabajo y la forma en que el individuo le da sentido pueden contribuir a un daño severo a la salud mental. Considerando el escenario actual, los profesionales de la salud están diariamente sujetos a eventos estresantes derivados de su proceso de trabajo. La investigación tiene como objetivo analizar las posibles implicaciones para la salud mental de los profesionales de la salud en el escenario pandémico causado por COVID-19. Para ello, el presente estudio se desarrolló a partir de una investigación bibliográfica, sustentada en investigaciones que abordaron el tema de la salud mental relacionada con las condiciones laborales de los profesionales de la salud en tiempos de pandemia. Del análisis de los datos se puede apreciar que el escenario actual y las demandas que enfrentan en su entorno laboral se establecen como factores contribuyentes para la aparición de daño emocional e implicaciones psicológicas para la salud de los profesionales de la salud. Así, los recursos de apoyo que ayuden en el acompañamiento psicológico de las demandas que experimentan estos profesionales son fundamentales en los servicios de salud.

Palabras clave: Salud Mental; Pandemia; Profesionales de la salud; Impactos en la salud.

\section{Introdução}

A saúde e o trabalho são reconhecidos como direitos sociais fundamentais conforme disposto na Constituição Federal brasileira de 1988 , em seu art. $6^{\circ}$. No que se refere aos direitos sociais, mais especificamente os direitos dos trabalhadores, o art. $7^{\circ}$ dispõe sobre a saúde do trabalhador, fruto de lutas ao longo da história até sua consolidação (BRASIL, 1988).

Ademais, somando-se a tal, destaca-se o direito à saúde da pessoa com transtornos mentais garantidos também pela Constituição Federal de 1988, através da Lei 10.216/2001, que assegura o bem estar mental, a integralidade psíquica e o pleno desenvolvimento intelectual e mental aos cidadãos (BRASIL, 1988).

Atualmente, o cenário de saúde mundial é marcado por diversas crises, sendo o enfrentamento a pandemia pelo COVID-19 a principal emergência de saúde pública enfrentada internacionalmente, uma vez que o surgimento e propagação do novo coronavírus, denominado de SARS-CoV-2, é responsável por milhões de mortes ao redor do mundo e inúmeras implicações nos diferentes âmbitos da vida do indivíduo (Dantas, 2021)

Os primeiros casos do novo coronavírus foram relatados em dezembro de 2019, na cidade de Wuhan, na China, se propagando rapidamente por todos os continentes. O Brasil teve seu primeiro caso diagnosticado em 25 de fevereiro de 2020 e atualmente, segundo a Organização Mundial de Saúde, já soma-se mais de 19 milhões de casos confirmados e mais de 500 mil óbitos (BRASIL, 2021)

Vale ressaltar que, desde o surgimento do novo coronavírus, visando o controle da propagação do vírus e a minimização do número crescente de óbitos, foram implementadas mundialmente estratégias de contenção que impactaram diretamente no 
cotidiano da população, resultando em implicações psicológicas relacionadas à COVID-19 (Aquino et al., 2020; Schmidt et al., 2020).

Dentro dessa perspectiva, a pandemia se configurou como um desafio global no que se refere à saúde mental, não só da população no geral, como também dos trabalhadores, sobretudo os profissionais de saúde, uma vez que são estes que lidam diretamente com eventos estressores relacionados às suas condições de trabalho, tais quais, fadiga e sobrecarga de trabalho; limitações de recursos e insuficiência de profissionais em virtude da superlotação; sentimentos de frustração e impotência pela exposição frequente aos eventos de mortes; os riscos ampliados de ser infectado no ambiente de trabalho, adoecer e morrer ou contrair o vírus e infectar familiares e et. (Schmidt et al., 2020).

Considerando o exposto, o presente estudo tem como objetivo analisar possíveis implicações na saúde mental dos profissionais de saúde no cenário de pandemia pela COVID-19, assim como, ampliar a discussão sobre a relação de trabalho e saúde mental e refletir perante os recursos de apoio em saúde mental adotados nesse contexto.

Dito isto, a relevância de pesquisar sobre o tema faz-se necessária uma vez que, apesar de tratar-se de um fenômeno recente, os estudos que se propõe a analisar a saúde mental dos profissionais de saúde em decorrência da pandemia pela COVID-19, configuram-se ainda, como escassos na literatura científica.

Ainda, em relação à metodologia adotada, tratar-se-á de uma pesquisa de cunho bibliográfico, sendo elaborada a partir de consultas em artigos de periódicos científicos, livros, teses e dissertações de mestrado e documentos emitidos pela Organização Mundial de Saúde, tendo como principais bases de dados a SciELO e o Google Acadêmico, com os seguintes descritores: Saúde Mental, Pandemia, Profissionais da saúde, Impactos na saúde.

\section{Relação do Trabalho e Saúde Mental}

O trabalho tem papel imprescindível na formação da identidade do sujeito e quando este é reconhecido e valorizado ganha importância significativa na vida do ser humano, de tal modo que o mesmo tem prazer em produzir e através da execução de suas tarefas tem a oportunidade de se realizar e constituir-se como um sujeito psicológico e social (Mendes \& Morrone, 2002).

Entretanto, estudos apontam que as condições de trabalho não satisfatórias estão ligadas diretamente com o surgimento de eventos que acarretam prejuízos ao bem-estar psicológico do trabalhador, ao não conseguir lidar com o enfrentamento das adversidades no contexto laboral (Ferreira, 2015).

Dejours (1992) ressalta em seus estudos, que quando as condições de trabalho são precárias somadas com a não contribuição com as regras determinadas pela organização do trabalho, isso resulta em riscos de acidentes e de aumento do sofrimento pela existência de sentimentos como ansiedade, medo e insatisfação.

Posto isto, o trabalho pode ser visto como um espaço fundamental para construção da identidade e da saúde, dado que "o lugar em que se desenrola para o sujeito a experiência dolorosa e decisiva do real, entendido como aquilo que -na organização do trabalho e na tarefa- resiste a sua capacidade, as suas competências, a seu controle" (Clot, 2007, p.59). Desse modo, compreende-se que o sofrimento mental ocorre quando o trabalho afeta diretamente os âmbitos de nossas vidas que são fontes geradoras e transformadoras de significados (Codo, Soratto, \& Vasquez-Menezes, 2002).

Nesse sentido, toda e qualquer atividade laboral tem papel atuante na saúde do trabalhador, e a forma como este significa suas vivências dentro do seu contexto de trabalho, pode repercutir tanto em prazer como em sofrimento.

Nessa perspectiva, as instituições de saúde reúnem situações adversas que expõem os trabalhadores ao acirramento do sofrimento psíquico, tais como, deparar-se constantemente com situações envolvendo morte, dor e sofrimento; a frequente sobrecarga de trabalho; a falta de preparo para lidar com demandas emocionais; a falta de reconhecimento que gera 
desmotivação e insatisfação no trabalho; dificuldades nas relações interpessoais; a insuficiência de recursos e a precarização do ambiente laboral (Borges, 2005).

Diante disso, muitos profissionais apresentam dificuldade em desenvolverem mecanismos próprios para lidar com as situações supracitadas, resultando no acometimento por uma alta carga de estresse e sentimentos de impotência e frustração (Kovacs, 2010).

O estresse, por si só, não se constitui capaz de desencadear uma disfunção ou doença, entretanto, a depender da sua exposição, de como e por quanto tempo as situações geradoras de estresse se mantém, pode representar riscos à saúde, contribuindo para a ocorrência de sofrimento e o surgimento doenças. Desse modo, o estresse pode ser compreendido como o resultado de uma relação particular entre o indivíduo, seu meio e as circunstâncias às quais encontra-se exposto (Farias \& Araújo, 2011)

Desse modo, o convívio constante e de forma intensa com situações como estas, pode acarretar no comprometimento da saúde mental do profissional, levando este a desencadear quadros de estresse ocupacional, como por exemplo, a síndrome de Burnout, tal conceito foi mencionado em 1974 como um fenômeno psicológico, pelo psiquiatra Herbet Freudenberger (Borges, 2005).

Nesse sentido, a definição de Burnout é compreendida como um fenômeno de cunho psicológico, resultante de uma "tensão emocional crônica, vivenciada pelos profissionais cujo trabalho envolve o relacionamento intenso e frequente com pessoas que necessitam de cuidado e/ou assistência” (Tamayo \& Tróccoli, 2002, p. 46).

Ainda, Borges (2005) apresenta a exaustão emocional, despersonalização e diminuição da realização pessoal como componentes relacionados à síndrome de Burnout, dessa forma, fenômenos como motivação no trabalho, significação do trabalho e condições (organizacionais e ambientais) do trabalho serão analisados em sua pesquisa como potenciais fatores de riscos.

\section{Impactos na Saúde Mental dos Profissionais de Saúde em Decorrência do Surgimento do Novo Coronavírus}

O coronavírus pode ser definido como uma família de vírus responsáveis por quadros de infecções respiratórias, os mesmos foram isolados pela primeira vez em 1937 e constatado como tal em 1965. Dentre essa família de vírus, destacam-se o SARS-COV, responsável pela síndrome respiratória aguda grave; o MERS-COV pela síndrome respiratória do Oriente Médio e o SARS-CoV-2, vírus que causa a doença coronavírus. disease 2019, mais conhecida como COVID-19 (Brasil, 2021).

Dito isto, o novo agente do coronavírus foi identificado em 31 de dezembro de 2019, após casos registrados na cidade de Wuhan, na China, rapidamente se propagando para os demais países do mundo, sendo decretado como uma crise pandêmica em 30 de janeiro de 2020 pela Organização Mundial da Saúde (Brasil, 2021).

Os diversos impactos psicológicos advindos da pandemia pelo novo coronavírus é explorado na literatura científica, como nos evidencia o estudo de Wang et al. (2020) desenvolvido com a população geral da China no estágio inicial da pandemia. O estudo constou com 1,210 participantes de 194 cidades e revelou que 28,8\% apresentaram sintomas moderados a severos de ansiedade, $16,5 \%$ sintomas depressivos e $8,1 \%$ de estresse.

Nesse sentido, os profissionais de saúde, que lidam diretamente com eventos estressores no seu ambiente de trabalho, também apresentaram significativos comprometimentos na saúde mental, uma vez que os desafios enfrentados na assistência à saúde dos indivíduos diagnosticados com COVID-19 podem ser um gatilho para desencadear ou até mesmo intensificar sintomas depressivos, ansiosos ou de estresse (Bao et al., 2020). 
Ademais, segundo Souza e Souza (2020) além do sentimento de medo e angústia de contrair o vírus enquanto exerce suas atividades laborais, os profissionais de saúde têm que lidar com desafios adicionais, como por exemplo, a frequente sobrecarga de trabalho; a escassez dos recursos materiais e humanos; incertezas sobre a eficácia dos métodos utilizados e preocupações com os riscos iminentes de contrair o vírus e infectar outras pessoas.

Pesquisas desenvolvidas com profissionais de enfermagem, destacam o alto nível de sofrimento psicológico dessa categoria, uma vez que os mesmos ocupam a linha de frente no combate ao COVID-19. Dito isto, segundo Yin et al. (2020) esses profissionais demonstraram riscos persistentes de desenvolver transtornos de estresse pós traumático (TEPT), mesmo após efetuar o afastamento periódico do ambiente trabalho.

Um estudo desenvolvido com médicos de Wuhan, na China, revela a forte pressão sofrida no ambiente de trabalho, o excesso de atividades, frustração, sentimento de exaustão, o que acarreta na ocorrência de problemas ligados à saúde mental que poderão refletir nas práticas adotadas e comprometer a capacidade na tomada de decisões, como por exemplo, sintomas depressivos, insônia, negação, raiva e insegurança (Kang et al., 2020).

\section{Recursos de Apoio em Saúde Mental e Intervenções aos Profissionais de Saúde}

Tendo em vista que a saúde é um direito fundamental de todo ser humano e garantido pela Constituição Federal Brasileira de 1988, é inegável a importância de possuir recursos necessários para lidar com as implicações na saúde mental em decorrência da configuração do atual cenário. Assim, uma série de serviços e intervenções constituem-se ferramentas relevantes ao acolhimento das queixas advindas da pandemia.

Os estudos recentes sugerem e reforçam a importância das ações de promoção e proteção da saúde mental dos profissionais de saúde, destacando a necessidade de dar mais visibilidade a temática e explorar esse conteúdo dentro do ambiente de trabalho, fornecendo suporte psicológico e estratégias visando minimizar a frequência dos danos à saúde mental dos profissionais daquele serviço (Texeira et al., 2020).

Tendo em vista os dados supramencionados, os profissionais da área da saúde mental, destacam a importância em resgatar práticas que proporcionam prazer, especialmente no contexto da pandemia, tais como, organizar a rotina de atividades diárias, ter cuidado com o sono, implementar práticas de atividades físicas e técnicas de relaxamentos no cotidiano, além de buscar maneiras para resgatar o vínculo e fortalecimento com a rede de apoio social (Banerjee, 2020).

As contribuições do psicológico durante a pandemia pela COVID-19 fazem-se de extrema necessidade para a população no geral e, sobretudo, aos profissionais de saúde, uma vez que propicia uma assistência em situações de crises, buscando o alívio dos sintomas ansiosos e ofertando apoio psicológico para lidar com os eventos emergentes e causadores de estresse (Schmidt et al., 2020).

Além do suporte comumente ofertado pelos serviços que compõem a Rede de Atenção Psicossocial às necessidades das pessoas em sofrimento mental, os atendimentos psicológicos de forma remota configuram-se como uma estratégia assertiva no contexto da pandemia, visto que tem demonstrado resultados relevantes e inúmeras vantagens na oferta de suporte emocional e psicológico, corroborando ainda, com as recomendações de distanciamento, quarentena e isolamento social (Schmidt et al., 2020).

Ainda, tecnologias de informações e comunicações ganharam evidências nesse contexto de pandemia, como os programas de Telessaúde, que oferta suporte online das mais diversas especialidades, através de soluções inovadoras de prestação de serviços em saúde durante o período de crise pela COVID-19 no Brasil (Cateano et al., 2020). 


\section{Considerações Finais}

Com base nos dados supramencionados nesse estudo, podemos constatar que o surgimento do novo coronavírus impactou significativamente a saúde mental da população no geral, em especial as pessoas que lidam diretamente com pessoas infectadas em seu cotidiano de trabalho, como é o caso dos profissionais de saúde.

Dessa maneira, reitera-se a importância de desenvolver um olhar sensível, cuidadoso e acolher perante os profissionais de saúde, uma vez que o ambiente que tem como propósito a promoção de modos de saúde acaba tornando-se gatilho de adoecimento e sofrimento mental a esses trabalhadores.

Para tal, o suporte psicológico alinhado aos serviços e ações em saúde mental soma-se como ferramentas imprescindíveis para a compreensão e superação de possíveis implicações psicológicas desenvolvidos no ambiente de trabalho, uma vez que a forma como esses sintomas são vivenciados nos serviços de saúde, poderão interferir diretamente nas habilidades e capacidades desses profissionais, além de progressivamente no seu bem-estar emocional.

Por tudo isto, entende-se que o estudo alcançou os objetivos propostos, ressaltando a importância de pesquisas adicionais sobre a temática, visando a qualidade do cuidado prestado aos profissionais de saúde e inovação das práticas em saúde mental no contexto de pandemia pela COVID-19 nos serviços públicos.

\section{Referências}

Aquino, E. M. L. et al. (2020). Medidas de distanciamento social no controle da pandemia de COVID-19: potenciais impactos e desafios no Brasil. Ciênc. saúde coletiva, Rio de Janeiro, v. 25, supl. 1.

Banerjee, D. (2020). The COVID-19 outbreak: crucial role the psychiatrists can play. Asian Journal Of Psychiatry, [S.L.], v. 5. Elsevier BV. http://dx.doi.org/10.1016/j.ajp.2020.102014.

Bao, Y. et al. (2020). 2019-nCoV epidemic: address mental health care to empower society. The Lancet, 395(10224), e37-e38. http://dx.doi.org/10.1016/S0140-6736(20)30309-3

Brasil. (1988). Constituição da República Federativa do Brasil. Brasília, DF: Senado Federal: Centro Gráfico. http://www.planalto.gov.br/ccivil_03/constituicao/constituicao.htm.

Brasil. (2021). Ministério da Saúde. Secretaria de Vigilância em Saúde. Boletim epidemiológico especial doença pelo coronavirus Covid-19. Brasília: Ministério da Saúde. https://www.gov.br/saude/pt-br/assuntos/boletins-epidemiologicos

Brasil. Ministério da Saúde. (2021). Coronavírus: o que você precisa saber e como prevenir o contágio. Brasília: Ministério da Saúde. https://saude.gov.br/saude-de-a-z/coronavírus.

Borges, L. O. (2005). Os profissionais de saúde e seu trabalho. São Paulo: Casa do Psicólogo.

Caetano, R. et al. (2020). Desafios e oportunidades para telessaúde em tempos da pandemia pela COVID-19: uma reflexão sobre os espaços e iniciativas no contexto brasileiro. Cadernos de Saúde Pública, [S.L.], 36(5). FapUNIFESP (SciELO). http://dx.doi.org/10.1590/0102-311x00088920.

Clot, Y. (2007). A função psicológica do trabalho. In: CLOT, Y. A função psicológica do trabalho. São Paulo: Vozes.

Codo, W., Soratto, L., \& Vasquez-Menezes, I. (2004). Saúde Mental e Trabalho. In: Zanelli, J. C.; Borges-Andrade, J. E.; Bastos, A. V. B. Psicologia, Organizações e Trabalho no Brasil Porto Alegre: Artmed.

Dantas, E. S. O. (2021). Saúde mental dos profissionais de saúde no Brasil no contexto da pandemia por Covid-19. Interface - Comunicação, Saúde, Educação, 25(1). FapUNIFESP (SciELO). http://dx.doi.org/10.1590/interface.200203.

Dejours, C. (1992). Trabalho e medo. In: Dejours, C. A loucura do trabalho. Cortez-Oboré.

Farias, M. D., \& Araújo, T. M. (2011). Transtornos mentais comuns entre trabalhadores da zona urbana de Feira de Santana-BA. Rev. Bras. Saúde ocup., São Paulo, 36(123).

Ferreira, A. P. (2015). Satisfação, sobrecarga de trabalho e estresse nos profissionais de serviço de saúde mental. Rev Bras Med Trab, [S.L], 2(13).

Kang L. et al. (2020). The mental health of medical workers in Wuhan, China dealing with the 2019 novel coronavirus. Lancet Psychiatry. 2020 Mar [acessado 30 Abr 2020]; 7(3):e14. https://pubmed.ncbi.nlm.nih.gov/32035030.

Kovács, M. J. (2010). Sofrimento da equipe de saúde no contexto hospitalar: cuidando do cuidador profissional. O Mundo da Saúde, São Paulo.

Mendes, A. M., \& Morrone, C. F. (2002). Vivências de prazer-sofrimento e saúde psíquica no trabalho: trajetória conceitual e empírica. In: Mendes, A. M.; Borges, L. O.; Ferreira, M. C. Trabalho em transição, saúde em risco. Brasília: EdUnB. 
Research, Society and Development, v. 10, n. 11, e596101119566, 2021

(CC BY 4.0) | ISSN 2525-3409 | DOI: http://dx.doi.org/10.33448/rsd-v10i11.19566

Schmidt, B., Crepaldi, M. A., Bolze, S. D. A., Neiva-Silva, L., \& Demenech, L. M. (2020). Saúde mental e intervenções psicológicas diante da pandemia do novo coronavírus (COVID-19). Estudos de Psicologia (Campinas), [S.L.], v. 37. FapUNIFESP (SciELO). http://dx.doi.org/10.1590/1982$0275202037 \mathrm{e} 200063$

Souza L. P. S., \& Souza, A. G. (2020). Enfermagem brasileira na linha de frente contra o novo Coronavírus: quem cuidará de quem cuida? J Nutr Health. https://periodicos.ufpel.edu.br/ojs2/index.php/enfermagem/article/ view/18444/11237

Tamayo, M. R., \& Tróccoli, B. (2002). Burnout no trabalho. In: Mendes, A. M.; Borges, L. O.; FerreirA, M. C. Trabalho em transição, saúde em risco. Brasília: EdUnB.

Teixeira, C. F. S et al. (2020). A saúde dos profissionais de saúde no enfrentamento da pandemia de Covid-19. Ciência \& Saúde Coletiva, [S.L.], 25(9). FapUNIFESP (SciELO). http://dx.doi.org/10.1590/1413-81232020259.19562020.

Yin, Q. et al. (2020). Posttraumatic stress symptoms of health care workers during the corona virus disease 2019. China: Clin Psychol Psychother. https://europepmc.org/article/med/3241 5733.

Wang, C. et al. (2020). Immediate psychological responses and associated factors during the initial stage of the 2019 coronavirus disease (COVID-19) epidemic among the general population in china. International Journal of Environmental Research and Public Health, 17(5), 1729.

http://dx.doi.org/10.3390/ijerph17051729. 